division of parts as has been described, is usually the only, or at least the chief, thing to be overcome. ${ }^{*}$ Lewis Sayre of New York divided an ordinary lacing boot into an anterior and posterior part, joining the separated portions of the sole by a ball-and-socket and of the uppers by indiarubber sheeting. Mr. Richard Davy followed close on the same lines. Both contrivances have many objections and inconveniences. My shoe consists of two thin steel plates

FIG. 5.

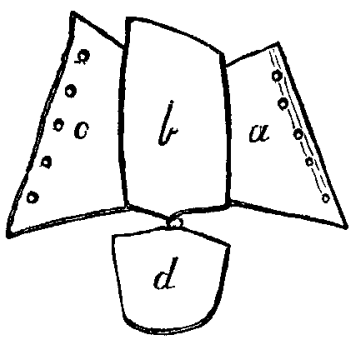

Sole and lappets of shoe jointed opposite the mid-tarsus

$a$ and $c$, Lappets with lace holes. $b$, Sole-piece.
$d$, Heel-plate. The lappet fastening the heel is omitted.

for the front and the back of the foot respectively, and each bent so as approximately to fit the curve of the part. They are jointed together by two loops or eyes that play within each other like the links of a chain. Both portions are enveloped in coutil or some such strong material, which continued on forms the lappets, which, on being laced, secure the appliance to the extremity (that to the heel portion is seen in Fig. 6). To the outer margin of the heel-plate is fixed an upright (simply a straight one in equinus) which carries atop the usual calf-plate. The upright is not intended to exercise any force and need only be very light. Riveted to the edges of the sole-plate is a very small eye or loop of steel. To apply turn the upright on its hinge back and out of the way, hold the heel and the sole-plate against those parts of the patient, bending the shoe in such direction

FIG. 6 .

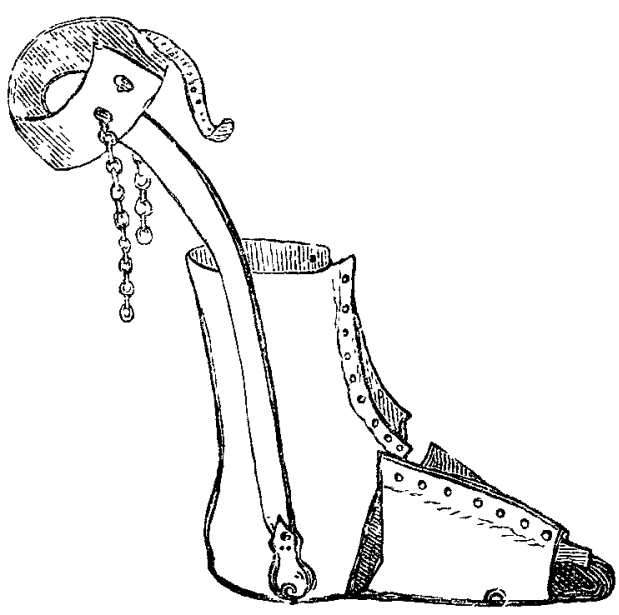

The Barwell shoe as it looks when applied. The upright is of the form intended for varus. 5

as will allow it to be laced on the heel portion first and with the plates lying fairly to the corresponding portions of the foot however distorted. Buckle the calf-piece in its place, tie a loop of cat-gut in the eye at the sole, and stretch between it and the chain at the calf-piece a sufficiently strong indiarubber spring. This shoe is applied outside the stocking and over it the patient can wear an ordinary walking shoe. With a little ingenuity the instrument can be almost entirely concealed. For calcaneus the great desideratum is to raise the os calcis. I do this by means of an accurately fitting sock (in some forms of foot a slit with lace holes is necessary) embracing the depressed heel and provided with an accessory part that passes over the neck and head of the astragalus. A piece of strong webbing is firmly sewn to the sock so that one end lies in the middle of the front border of the heelpiece, is stitched beneath it, and also to the back where it

4 Scarpa's shoe, no doubt a creditable instrument at the time of its invention, is faulty in many ways besides the oneness of the sole.

5 For equinus the upright should be straight. If much varus twist also present one eye on the outer side of the sole-plate is required ; if the equinus be pure and without varus, as in Fig. 1, an eye on each side is necessary. continues to be sewn to the sock for an inch or more, lying thus for that part of its course behind the Achilles tendon. This appliance is put on over a well-fitting stocking (silk is best) and inside the out-door shoe, which only differs from an ordinary shoe by having a round socket right through the heel from side to side. Into this socket; is

FIG. 7.

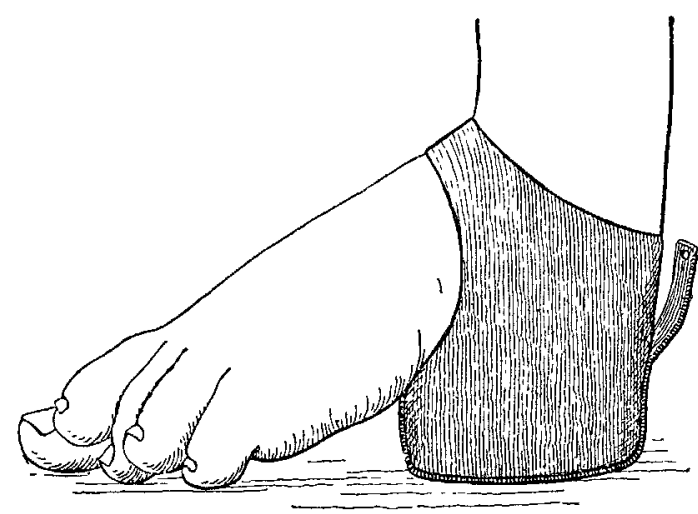

Calcaneus percavus with sock applied.

secured, but so as to permit free movement to the foot, either a single or double very slight upright with calf-plate. The webbing at the back of the sock is connected by an accumulator to eyes, where the uprights and calf-plate meet or, in some cases, by one on each side. The power thus applied not only draws up the heel, but also, by means of the band running over the instep, depresses the abnormally raised head of the astragalus. In the form of the distortion termed "sursum flexus" the lifting of the heel thus effected aids progression in that it brings the front of the foot also into better position. In cavus, such as is shown in Figs. 3 and 4, the toes are sometimes sufficiently depressed to cause the patient to trip. This may be obviated by an indiarubber spring going from the calf-plate to a lappet on the front of the boot. These mechanisms are very valuable each in its own special case. In using them it must be remembered that the spring force is not intended to rectify the deformity all at once, but by gradual influence more or less rapid or more or less slow, as the case may be.

Wimpole-street, $W$

\section{THE TELEPHONE AND ITS APPLICATION TO THE DEAF.}

BY BERTRAM THORNTON, L.R.C.P. LOND., M.R.C.S. ENG.,

SLRGEOX TO THE ROYAL SEA BATHING INFIRMARY AND MEDICAX OFFICER TO THE DEAF AND DUMB ASYLUM, MARGATE.

THE remarkable advances in the application of electricity to the daily requirements of mankind, and more especially the wonderful invention of the telephone, have doubtless stimulated many scientific minds to devise some apparatus to assist the deaf to hear. It has been stated that $\mathrm{Dr}$. Bell, the inventor of the telephone, in his efforts to contrive an electrical instrument for this purpose, hit upon the marvellous contrivance which now plays such a large share in the social and mercantile circles of all civilised countries.

Up to the present time there is no instrument prominently before the profession which on the lines of the telephone is intended to assist the hearing of deaf persons. It is with no wish to claim originality or any special knowledge of electricity, that I desire to call attention to one of the more recent moulifications of the telephone which was brought to my notice in the summer of 1894 . Through the courtesy of the manager of the General Electric Company 1 had the opportunity of investigating the powers of this instrument, and I soon came to the conclusion that it was likely to be of material service for deaf people if it could be adapted to their special requirements. Further experience confirmed my original opinions and a considerable portion of my spare time has been spent in testing its powers on deaf mutes and deaf patients and in contriving suitable handles, switches, \&c., in order to adapt it to the peculiar needs of the deaf. As medical officer to the Margate Branch of the Deaf and Dumb Asylum, Old Kent-road, with the charge of 300 deaf mutes, my first thought was to find out whether the 
instrument could be made of service in thcir education. 'The head master, Dr. Elliott, cordially fell in with my desire and bas given me valuable assistance. In investigations of this sort one is met by the initial difficulty that there is no convenient method of estimating the volume and intensity of sound. Inciclentally one would call the attention of scientists to the need of a practical "sonometer" or "phonometer," in the hope that an inventor may be stimulated to design such a desirable instrument. The usual methods of testing the hearing powers by means of whistles, bells, musical instruments, the ticking of watches, and tuning forks are both clumsy and unscientific. What is required is an inexpensive instrument contrived on scientific lines, with a definite and easily applicable unit of sound. It is, perhaps, unnecessary to allude to the care that must be taken in testing the hearing powers of deaf mutes with the tuning fork and other methods ; it is most difficult to make a deaf-mute child distinguish between "sound" as appreciated by the auditory nerve and "vibration" as felt by the sensory nerves. After all, the human voice is perhaps the most useful instrument in testing the hearing powers of very deaf persons for educational purposes. If a deaf mute cannot be made to hear the voice of his teacher with or without the assistance of some mechanical contrivance, the results obtained by tuning forks, \&c., fade into a question of merely clinical interest.

It is no part of my intention to add to the length of this paper by quoting the statistics of asylums for the instruction

FIG. 1.

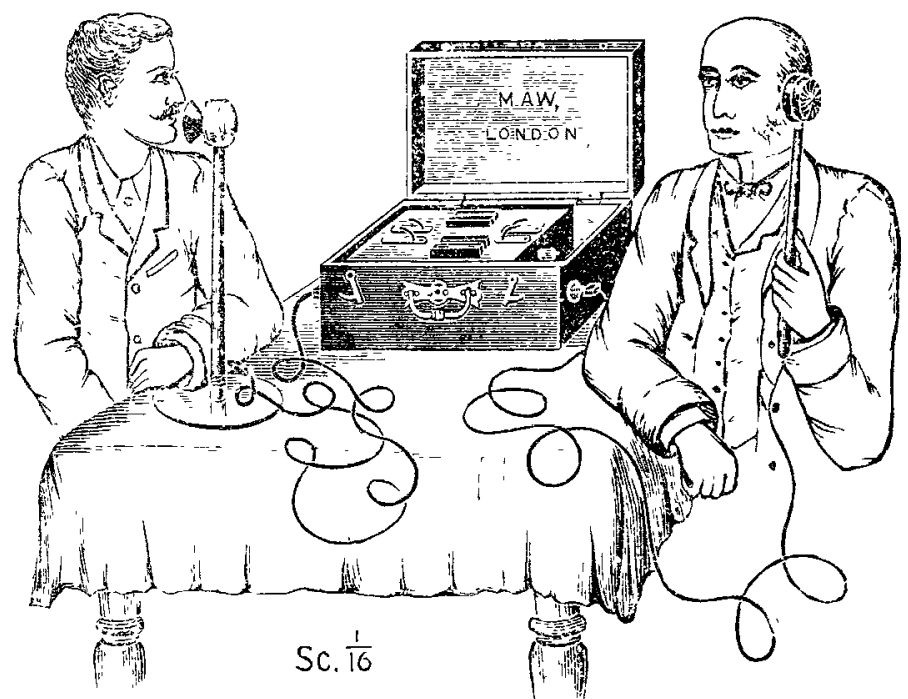

Shows the instrument in use. The transmittor would usually be leld in the hand, detached from its base. It is not necessary for the speaker's mouth to be so close to the instrument as shown in the slietch.

of the deaf; those interested in the subject should consult among other works those of Hartmann, St. John Roosa, Mygind (1894), and Love and Addison (1896). In every institution for the education of deaf mutes a certain proportion of the children possess some degree of hearing; it would not perhaps be over-estimating the percentage to say that, speaking generally, from 10 to 20 per cent. of these children are capable of hearing with or without instrumental aid vowels or words and sentences. The less fortunate ones must rely entirely on lip reading or on the sign system of education. The investigations I carried out with the assistance of Dr. Elliott at the Margate Asylum showed that the telephone promised to be of material assistance in at least from 10 to 15 per cent. of the children. For some years past an excellent modification of the ordinary binaural stethoscope with a metal bell-shaped mouthpiece has been used at the Margate Deaf and Dumb Asylum for all children with any useful degree of hearing; this instrument was specially devised by Mr. Golding-Bird, the consulting surgeon to the asylum. In using this contrivance it is necessary for the mouth of the speaker to be almost in contact with the bell-shaped mouthpiece. With the ordinary speaking-tubes and trumpets it is also essential that the mouth of the speaker should be clirected into, and closely applied to, the orifice of the instrument; by this means it is well known that the intensity of the voice of the speaker is increased to an almost painful degree, but the effort of keeping the mouth applied immediately to the mouth of the tube or trumpet prevents the hearer from witnessing the movements of the lips and general expression of the speaker-a most important consideration with deaf people.

With the telephone I found that one could produce an intensification of the voice at least equal to the most approved form of ear-trumpet at a ristance varying from six inches to a foot from the mouth of the transmitter; by approaching the mouthpiece to within two or three inches the sound was obviously distressing to some of these deaf mutes. At a distance of a foot from the mouth of the transmitter we were able to make some of the best "hearing children" easily hear sentences of filmiliar words spoken in a low conversational voice, the speaker being inside a room and the child outside with the door shut. In testing deaf and dumb children it is, of course, essential to use words whose sound is familiar to them; if words are used whose sound is unfamiliar they are unable to reproduce them either by speech or writing; in the same way a person with normal hearing would be unable promptly to repeat a sentence of some unknown language. With the telephone as well as with other instruments that assist the hearing powers vowels are obviously more clearly heard than consonants, but there is this important advantage with regard to the telephone that the speaker has not to approach his lips so close to the transmitter, consequently the listener is more easily able to read the lip and lingual movements and observe the expression of the speaker's face. In the great majority of the children I examined at the

Fig 2.

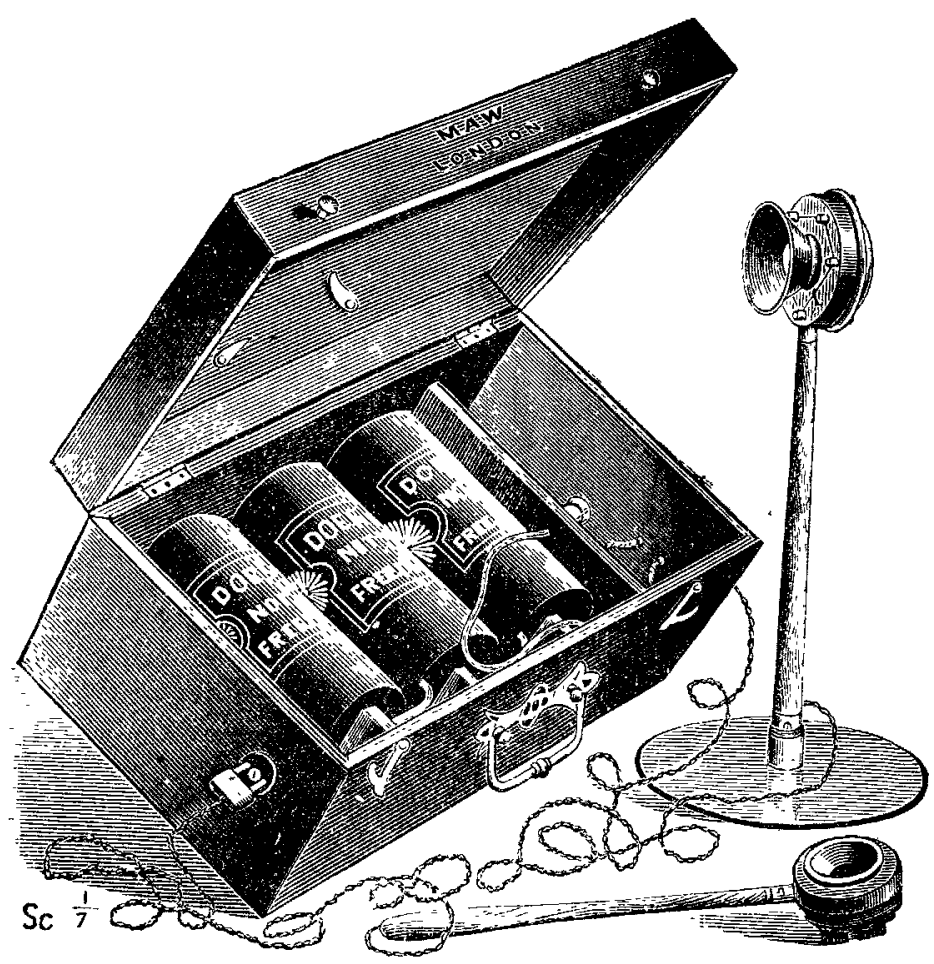

Shows (1) the case with three dry cells, wires, and connectingplug; (2) the receiver lying down; and (3) the transmittor standing up on its detachable stand.

asylum with any hearing power at all it was found that the tuning fork was not heard in contact with the forehead, but in every instance it was clearly heard when the base of the vibrating fork was placed in contact with the mouthpiece of the transmitter, and in several cases the vibrations were heard for a considerable period. A larger proportion of the above-mentioned children were able to hear the tuning fork vibrating one inch distant from the ear (not in contact), but with one or two exceptions all of them heard the vibrations when the fork was held within an inch of the mouthpiece of the transmitter. In these experiments every care was taken not to confuse auditory sensation with tactile sensation.

By the use of this instrument, the receiver being closely held to the listener's ear, the sound of the speaker's voice is reproduced opposite to, and within an inch and a half of, the membrana tympani; the sound waves being confined in the external meatus there is no loss by radiation. To get the best results it is necessary for the speaker to speak clearly, distinctly, and without unduly raising the voice. Excessive loudness of the voice or too close an approximation of the speaker's mouth to the transmitter painfully increases the intensity of the sound, and at the same time the clearness of the words is impaired or altogether obscured. My experience tends to show that this modification of the telephone promises to be of material use in the education of those deaf mutes who possess a fragment of hearing power, and it has the following important advantages over the single speaking tube that is sometimes used: firstly, that the wires from several receivers can be coupled 
up to one transmitter, and thus a teacher can instruct a group of children at the same time; and, secondly, that, as it is not necessary for the teacher to apply his mouth close to the transmitter, the pupils have a full view of his facial expression and lip movements, which is not the case when he has to direct his attention and his voice into the mouth of a speaking tube or trumpet.

In the experiments I have made with patients suffering from lesser degrees of deafness than the deaf-mute children at the Margate Asylum the utility of the telephone has been proportionately more apparent. I will mention two cases in illustration. The first was that of a young woman with nerve deafness so extreme that it was necessary to speak at the top of one's voice within two or three inches of her ear to enable her to hear, but with the telephone I was able to converse in a subdued voice with my mouth twenty-six inches away from the transmitter; in this caso she received some assistance from reading my lips, though she was only a beginner in this accomplishment. The second case, a man with a similar degree of deafness, was able to hear his wife and myself conversing with him in tones hardly raised above a whisper, we being inside a room and he being outside with the door shut. I venture to give these two cases instead of quoting tuningfork statistics because they convey approximately some notion of the possibilities of this instrument.

Whether future experience will bear out my preliminary investigations only time will show; the opportunities of a provincial surgeon for carrying out extensive scientific observations are naturally limited. In addition to much kind help from Mr. Max Binswanger of the General Electric Company I have received valuable assistance from Messrs. Maw, Son, and Thompson, who have skilfully carried out my suggestions as regards providing the instrument with appropriate-shaped handles, dry-cell battery, and case. The latter firm will be glad to show the apparatus to anyone interested in the matter. I understand that the cost of the instrument will place it within the power of purchase of persons of moderate means. Like all electrical contrivances it has the inevitable drawback of a battery and wires. It will consequently be suited to the more stationary requirements of the domestic hearth and the dinner table; in the church, theatre, or in courts of justice it could be easily fitted up at trifling expense for the convenience of persons with defective hearing. My friend Dr. Benson of Maida-vale has suggested

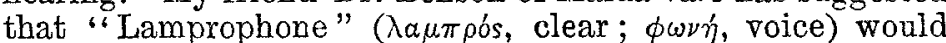
be an appropriate name in the event of the instrument proving itself to possess sufficient practical utility to warrant it requiring a special term to describe it. In conclusion, I wish to disclaim any special originality as an inventor; but in my attempt to adapt other men's ideas for the benefit of deaf people I trust that I may have done some small service, even if this paper only serves to stimulate others to carry my investigations to a more perfect issue.

Margate.

\section{THE INFLUENCE OF GLYCERINE IN CULTURE MEDIA ON THE DIPHTHERIA BACILLUS.}

BY A. M. GOSSAGE, M.B. OxON.,

ASSISTANT PHYSICIAN TO THE WESTMINSTER HOSPITAT.

[From the Westminster Bacteriological Laboratory.]

DURING the course of an inquiry into the life-history of the diphtheria bacillus outside the body I was leacl, owing to a kind suggestion by Dr. Blaxall, to try the effect of cultivating it on media to which glycerine had been added in varying proportions. It has long been known that the Klebs-Löller bacillus will grow more profusely on nutrient agar-agar to which 5 or 6 per cent. of glycerine has been added than on ordinary rutrient agar-agar. Dr. Kanthack and Mr. Stephens, moreover, in a paper read before the Pathological Society on Jan. 21st, recommended a culture medium containing alkalinised ascitic fluid, agar-agar and 5 per cent. of glycerine, and considered this medium to have a strong selective action on the Klebs-Lüfller bacillus. In view of this apparently favourable influence of glycerine it seemed not unlikely that the addition of larger proportions of glycerine might make culture media still more selective for the bacillus of diphtheria. On nutrient agar-agar media, to which from 12 to 15 per cent. of glycerine had been added found that those organisms which are commonly ob tained when a serum tube is infected from a throat grew decidedly less readily than the diphtheria bacillus. Agar-agar tubes, containing varying proportions of gly cerine between 6 and 15 per cent., were infected at the same times and with the same materials from, cases of diphtheria as tubes containing serum or Löfler's serum, and thus impure cultures of the Klebs-Lüfter bacillus were obtained. The cultures on the media containing the largest proportion of glycerine were always the least impure, although the employment of nutrient agar agar containing 18 per cent. of glycerine did not completely eliminate the contaminating micro-organisms. These large proportions of glycerine seemed to be specially unfavourable to the bacillary forms of these micro-organisms, although torule, sarcine, and cocci all appeared to grow less readily than the diphtheria bacillus. The addition of at least 9 per cent. of glycerine was required to get a less impure growth than that obtained on serum; indeed, when only 6 per cent. was addec the growth was usually more impure than that on serum. My experience with the medium recommended by Dr Kanthack and Mr. Stephens was not favourable. I have made twenty separate comparisons between this medium and serum by infecting tubes of each at the same times with the same swabs from the throats of diphtheria cases. In all except one instance the growth after twentyfour hours of the Klebs-Loffler bacillus was more impure on this medium than on serum. A further objection to this medium was found in the fact that the bacilli grown on it did not stain so readily with methylene blue as those grown on serum.

The practical value of the selective action of nutrient agaragar media, containing large percentages of glycerine, on the Klebs-Löffler bacillus was much discounted by the very small total growth obtained after twenty-four hours' incuba. tion, where the proportion of glycerine present was $12 \mathrm{per}$ cent. or higher. A trial was, therefore, made with serum to which 6,10 , or 12 per cent of glycerine bad been added. By this means beautifully clear media were obtained, which seemed to require heating to a degree or two higher than ordinary serum in order to induce firm setting. With these media very similar results were obtained to those with glycerine agar-agar. When 6 per cent. of glycerine had been added the resulting growth twenty-four hours after infection: from a case of diphtheria was about the same as on ordinary serum, and there seemed to be no more selective action on the Klebs-Löffler bacillus than with serum. With the employment of higher percentages of glycerine there was. apparently a decided selective action, but the total growth was proportionately diminished. In two cases apparently pure growths were obtained on 10 per cent. glycerine serum, whilst the cultures on serum and the KanthackStephens medium, obtained by infection with the same materials at the same times, were very impure from the presence of other bacilli and cocci. On the other hand, however, on two occasions streptococci were found in the: cultures on 12 per cent. glycerine serum, while the growths. on serum and the Kanthack-Stephens medium were free from this impurity.

A more promising result from my experiments than this selcctive action was the manner in which the individual bacilli stained with Löffler's alkaline methylene blue after growth for twenty-four hours on these glycerine-containing media. The bacilli showed a remarkable afinity for the stain and metachromatism was very conspicuous. After growth on glycerine serum each bacillus showed, when stained, a deep violet dot at each pole, with frequently one or more dots in between, whilst the remainder of the bacillus was colourless or faint blue. The other micro organisms in specimens from impure cultures were nearly always stained blue, the only exception being that occasionally a few cocci took up the violet colouration. This staining result was invariable in twenty-one instances in which specimens were made from glycerine scrum cultures obtained from cascs of diphtheria, and in each case all the diphtheria bacilli were so stained. A similar constant result was obtained with pure cultures on glycerine serum and cultures obtainea from two guinea-pigs that had been infected with diphtheria. Specimens from growths on agar-agar to which 9 or more per cent. of glycerine had been added stained with methylene blue in exactly the same way, but the bacilli were more irregular in shape and size than those grown on glycerine serum; some were remarkably large and thick even in 